\begin{tabular}{|c|c|c|}
\hline & Int.J.Curr.Microbiol.App.Sci (2016) 5(10): 424-431 & \multirow{2}{*}{ 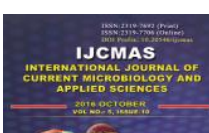 } \\
\hline & \multirow{4}{*}{$\begin{array}{l}\text { International Journal of Current Microbiology and Applied Sciences } \\
\text { ISSN: 2319-7706 Volume } 5 \text { Number } 10(\mathbf{2 0 1 6 )} \text { pp. 424-431 } \\
\text { Journal homepage: http://www.ijcmas.com }\end{array}$} & \\
\hline & & \\
\hline EXCELLENT & & \\
\hline PUBLISHERS & & wwwi.jemas.con \\
\hline
\end{tabular}

Original Research Article http://dx.doi.org/10.20546/ijcmas.2016.510.048

\title{
Characteristics of Blood Stream Isolates in Urosepsis from a Tertiary Care Hospital
}

\author{
Martha Rinnah Bijou, K. Sandhya Bhat* and Reba Kanungo \\ Department of Microbiology, Pondicherry Institute of Medical Sciences, India \\ *Corresponding author
}

\begin{tabular}{|c|c|}
\hline & A B S T R A C T \\
\hline & \multirow{7}{*}{$\begin{array}{l}\text { Urosepsis refers to clinically evident severe infection of the urinary tract along with features } \\
\text { of bacteremia. Urosepsis in adults comprises approximately } 25 \% \text { of all sepsis cases with } \\
\text { high mortality rate of } 25-60 \% \text {. This study was conducted to determine the bacteriological } \\
\text { profile and antimicrobial susceptibility pattern of the organisms associated with urosepsis. } \\
\text { A descriptive study was carried out after obtaining approval from Institute Ethics } \\
\text { Committee. Demographic details, duration of hospital stay, underlying risk factors, } \\
\text { bacteriological profile and antimicrobial susceptibility pattern of the isolates, were recorded } \\
\text { from } 52 \text { patients confirmed to have urosepsis. Statistical analysis was done by using IBM } \\
\text { SPSS software. Overall incidence of urosepsis was } 3.8 \% \text { in patients admitted to } \\
\text { the hospital during the study period. Type } 2 \text { diabetes mellitus was the major risk factor } \\
\text { (71\%), with underlying renal condition/s. Escherichia coli was the predominant isolate } \\
(75 \%) \text {. Organisms showed highest resistant against ampicillin (95.3\%); least resistance was } \\
\text { observed for imipenem ( } 2.2 \%) \text {. This study highlighted the bacterial profile with their } \\
\text { susceptibility pattern, thus paving a way for formulating antibiotic policy for empiric } \\
\text { treatment of urosepsis. Documentation of associated risk factors would help in taking } \\
\text { preventive measures to decrease morbidity and mortality. }\end{array}$} \\
\hline Keywords & \\
\hline & \\
\hline & \\
\hline $\begin{array}{l}\text { Escherichia coli, } \\
\text { Antibiogram. }\end{array}$ & \\
\hline Artic & \\
\hline $\begin{array}{l}\text { aber } 2016 \\
\text { Online: } \\
\text { re } 2016\end{array}$ & \\
\hline
\end{tabular}

\section{Introduction}

Urosepsis implies to a clinically evident severe infection of the urinary tract associated with features of systemic inflammatory response syndrome or bacteremia (Sorensen et al., 2013; Kalra et al., 2009). It is postulated that ascending urinary tract infection (UTI) from the bladder to the kidney, with resultant bacteremia is the main cause of urosepsis (McNally et al., 2013). Urosepsis in adults comprises approximately $25 \%$ of all sepsis cases following an episode of complicated urinary tract infection (Wagenlehner et al., 2013; Lipsky et al., 1989). Gram-negative rods (75-85\%), are most commonly associated with this condition; while grampositive organisms are less frequently (15\%) involved (Gosciniak et al., 2014; Wagenlehner et al., 2008).

Elderly patients, patients with uncontrolled diabetes mellitus, patients on dialysis, renal transplant recipients and those on chemotherapy or prolonged course of steroids are at increased risk of developing urosepsis (Garg et al., 2015; Wagenlehner $e t$ al., 2007). Urosepsis must be detected at an early stage and promptly treated with 
appropriate antimicrobial agents to prevent complications. Successful management of urosepsis depends on early specific therapy, maintaining optimal concentrations of antimicrobial agents both in the blood and urinary tract. It also requires control of factors leading to complications including any septic foci in the urinary tract (Hofmann, 1990 and Geerlings et al., 2000). Studies have shown antimicrobial resistance to be common in complicated UTI leading to poor response to therapy (Tal et al., 2005; Cardoso et al., 2008). Several studies have documented resistance pattern of organisms in UTI; however, there is lack of data on urosepsis. Hence, this study was undertaken to, describe the bacteriological profile and the antimicrobial susceptibility pattern of the organisms associated with urosepsis and determine the risk factors in patients admitted to a tertiary care hospital.

\section{Materials and Methods}

The descriptive study protocol was reviewed by the Institute Ethics Committee and approved. (RC No- RC/14/139). Retrospective data of all patients diagnosed to have urosepsis by simultaneous positive urine and blood culture during the period from June 2014 to July 2015, were analysed from hospital and laboratory records. A total of 52 patients with urosepsis fitted into the criteria. A similar antibiogram was taken as a method to link the urine and blood isolate. Repeat isolates from the same patients, isolates with differing antibiogram and patients with incomplete case records were excluded from this study. The demographic details, risk factors, duration of fever, length of hospital stay, treatment history and the outcome of each patient, along with bacteria isolated and its antibiogram were recorded in all 52 cases.

The antimicrobial agents tested by standard Kirby Bauer disc diffusion technique for all gram-negative bacterial isolates were ampicillin $(10 \mu \mathrm{g})$, piperacillin $(100 \mu \mathrm{g})$, amoxicillin+clavulanate $(20 / 10 \mu g)$, piperacillin/ tazobactum $(100 / 10 \mu \mathrm{g})$, gentamicin $(10 \mu \mathrm{g})$, amikacin $(30 \mu \mathrm{g})$, cefotaxime $(30 \mu \mathrm{g})$, ceftriaxone $(30 \mu \mathrm{g})$, ceftazidime $(30 \mu \mathrm{g})$, ceftazidime+clavulanate $(30 / 10 \mu \mathrm{g})$, cefoperazone+sulbactam $(75 / 10 \mu \mathrm{g})$, cefoxitin $(30 \mu \mathrm{g})$, ciprofloxacin $(5 \mu \mathrm{g})$, levofloxacin $(5 \mu \mathrm{g})$, imipenem $(10 \mu \mathrm{g})$, meropenem (10 $\mu \mathrm{g})$ Penicillin (10 units), amoxicillin+clavulanate $(20 / 10 \mu \mathrm{g})$, erythromycin $(15 \mu \mathrm{g}), \quad$ clindamycin $(2 \mu \mathrm{g})$, ciprofloxacin $(5 \mu \mathrm{g})$, amikacin $(30 \mu \mathrm{g})$, gentamicin $(10 \mu \mathrm{g})$, cefoxitin $(30 \mu \mathrm{g})$, cotrimoxazole $(25 \mu \mathrm{g})$, teicoplanin $(30 \mu \mathrm{g})$, and linezolid $(30 \mu \mathrm{g})$ were tested for all grampositive isolates.

Oxacillin screen agar and cefoxitin disk diffusion tests were used for screening for methicillin resistance and vancomycin screen agar for screening vancomycin resistance among gram-positive bacterial isolates (Pradipta et al., 2013).

Gram-negative bacterial isolates were also recorded as Extended Spectrum $\beta$ lactamases (ESBL) or non ESBL producers by noting down their susceptibility results to cefotaxime $(30 \mu \mathrm{g})$, ceftriaxone $(30 \mu \mathrm{g})$, ceftazidime $(30 \mu \mathrm{g})$. Further these isolates were confirmed as ESBL producers by recording the results of the phenotypic confirmatory disc diffusion test (PCDDT), using ceftazidime (30ug) and ceftazidime + clavulanate $(30 \mu \mathrm{g} / 10 \mathrm{ug})$ disc. An increase of $\geq 5 \mathrm{~mm}$ in the zone of inhibition of the combination discs in comparison to that of the ceftazidime disc alone, was considered to be a marker for ESBL production (CLSI 2015). Multidrug resistance was documented as organisms showing resistance to more than three classes of antibiotics (Magiorakos et al., 2012). Statistical analysis was done by using IBM SPSS software. 


\section{Results and Discussion}

Out of 5,599 clinically suspected patients with urinary tract infection, $1,372(24.5 \%)$ had culture positive UTI, during the study period. Out of these $52(3.8 \%)$ patients were diagnosed to have urosepsis and were included in the study. Maximum patients (46.2\%) were $\geq 60$ years, with mean age of the study population was $60.21 \pm 13.525$ years with $65.4 \%$ males. Male: female ratio was 3:2. Majority of the patients had a hospital stay of more than one week for up to one month, with mean duration of $13.45 \pm$ 10.486 days.

Type 2 diabetes mellitus was the major risk factor accounting for $71 \%$ with one or more underlying renal condition, as additional contributing factor/s. Among them, acute kidney injury was most commonly seen $(40 \%)$, followed by chronic kidney disease (30\%), hydroureteronephrosis (6\%), pyelonephritis (9\%) and 3\% each with acute renal failure, acquired cystic kidney disease, post-renal transplant acute graft rejection, papillary necrosis and renal abscess. Chronic liver disease and bronchial asthma were also noted in some patients. Distribution of patients with urosepsis based on underlying co-morbidities is shown in figure 1.

Single organism was isolated from all 52 cases. Distribution of blood stream isolates in 52 patients with urosepsis is shown in figure 2. None had polymicrobial infection.

Antimicrobial resistance rate observed in our study, in the decreasing order was as follows: ampicillin (95.3\%), piperacillin (77.3\%), cefotaxime $(75 \%)$, amoxicillin +clavulanate (69.2\%), ciprofloxacin (68\%), ceftriaxone (52.3\%), meropenem (51.2\%), gentamicin (26.5\%), cefoxitin (15.3\%), cefoperazone+sulbactam (15\%), piperacillin +tazobactam (12.5\%), amikacin (4.1\%) and imipenem (2.2\%). Antimicrobial resistance pattern of gram-negative bacterial isolates from patients with urosepsis is shown in table 1 and figure 3 .

Resistant to at least one agent in three or more antimicrobial categories (multi drug resistance) was noted in 26 isolates. Distribution of 22 multidrug resistant gramnegative bacteria among urosepsis isolates is shown in figure 4 .

Six out of 22 E. coli, were found positive for the production of Extended Spectrum $\beta$ Lactamase (ESBL).

Among the few gram-positive organisms isolated (3 in number), Methicillin Sensitive Staphylococcus aureus (MSSA) was susceptible to penicillin and all other betalactam antibiotics as well as aminoglycosides and fluoroquinolones. On the other hand a single isolate of Enterococcus faecalis was multidrug resistant and susceptible to linezolid, vancomycin and teicoplanin.

Urosepsis resulting from complicated UTI in patients with underlying risk factors increases morbidity and mortality in these patients. Successful management of patients with urosepsis depends on isolating the causative agent and instituting specific antibiotic therapy, along with removing the focus of infection (Sugimoto et al., 2013).

In the current study, urosepsis was noted in $3.8 \%$ patients, among 1,372 culture positive cases with UTI and $46.2 \%$ were above the age of 60 years with a male preponderance. Urosepsis was reported in all age groups regardless of gender by Kalra et al., 2009. They found the infection to be more common among patients with underlying comorbidities. Similar observations were also made in the present study with $76.4 \%$ patients had one or more underlying renal 
condition/s. UTI in males is usually associated with complications; with greater chance of developing urosepsis. Prostatic hypertrophy is an additional contributing factor due to urinary obstruction (Kalra et al., 2009; Lipsky, 1989).

Majority of the patients (71.2\%) had type 2 diabetes; similar to a study by Garg et al., 2015, where uncontrolled diabetes mellitus was associated with pyelonephritis further leading to urosepsis. Studies have shown that prolonged uncontrolled diabetes can lead to changes in the genitourinary system leading to severe complicated UTI.

Acute kidney injury, chronic kidney disease, hydroureteronephrosis, pyelonephritis and acute renal failure, acquired cystic kidney disease, post-renal transplant acute graft rejection, papillary necrosis and renal abscess were some of the risk factors contributing to urosepsis in the present study. Sorensen et al., 2013 showed a significant fraction (32\%) of patients with urosepsis had clinically important radiological findings, indicating that an abnormality of the kidney or the urinary tract could predispose to complicated UTI; hence leading to urosepsis. Therefore, imaging of the urinary tract in adult patients with an acute upper UTI with associated bacteremia could help in detecting the underlying pathology (Sorensen et al., 2013).

Gram negative organisms predominantly, $E$. coli followed by Klebsiella have been reported in most of the studies including the present study (Chen et al., 2013; Schweizer et al., 2011). Pseudomonas is associated with long term hospitalization. Multidrug resistance to commonly used antibiotics like fluroquinlones, aminoglycosides and combination antibiotics like piperacillin/ tazobactam or amoxicillin/ clavulanic acid, have been increasingly reported in various studies including present study (Magiorakos et al., 2012; Chen et al., 2013).

Table.1 Antimicrobial agents tested for gram-negative bacterial isolates and their resistance pattern

\begin{tabular}{|l|l|l|}
\hline Antimicrobial Agent & No. of isolates tested & Resistance (\%) \\
\hline Ampicillin & 43 & 95.34 \\
\hline Piperacillin & 22 & 77.27 \\
\hline Amoxicillin+clavulanate & 26 & 69.23 \\
\hline Piperacillin+tazobactam & 48 & 12.5 \\
\hline Gentamicin & 49 & 26.53 \\
\hline Amikacin & 49 & 4.08 \\
\hline Ceftriaxone & 42 & 52.38 \\
\hline Cefotaxime & 40 & 75 \\
\hline Cefoxitin & 26 & 15.38 \\
\hline Cefoperazone+sulbactam & 40 & 15 \\
\hline Levofloxacin & 30 & 20 \\
\hline Ciprofloxacin & 50 & 68 \\
\hline Imipenem & 45 & 2.22 \\
\hline Meropenem & 43 & 51.16 \\
\hline
\end{tabular}


Fig.1 Distribution of patients with urosepsis based on underlying co-morbidities

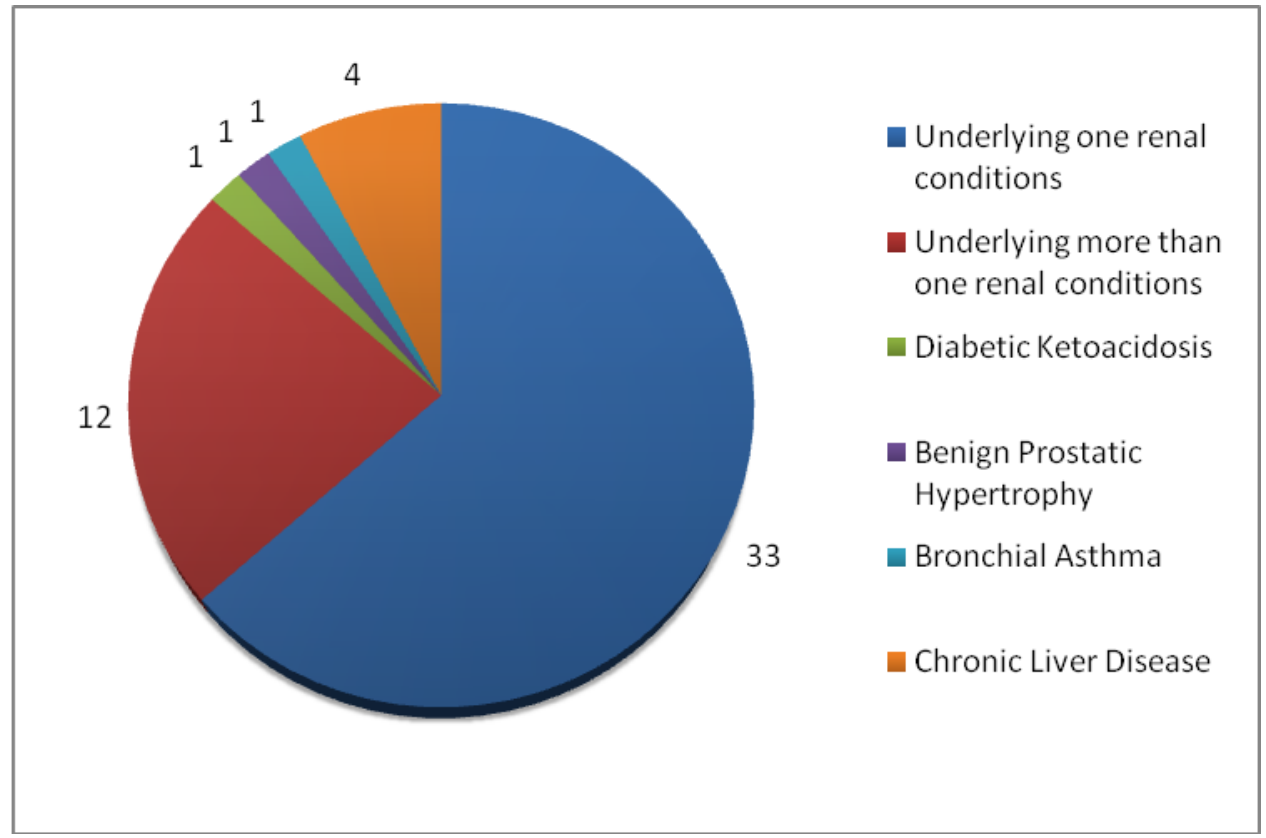

Fig.2 Distribution of blood stream isolates in 52 patients with urosepsis

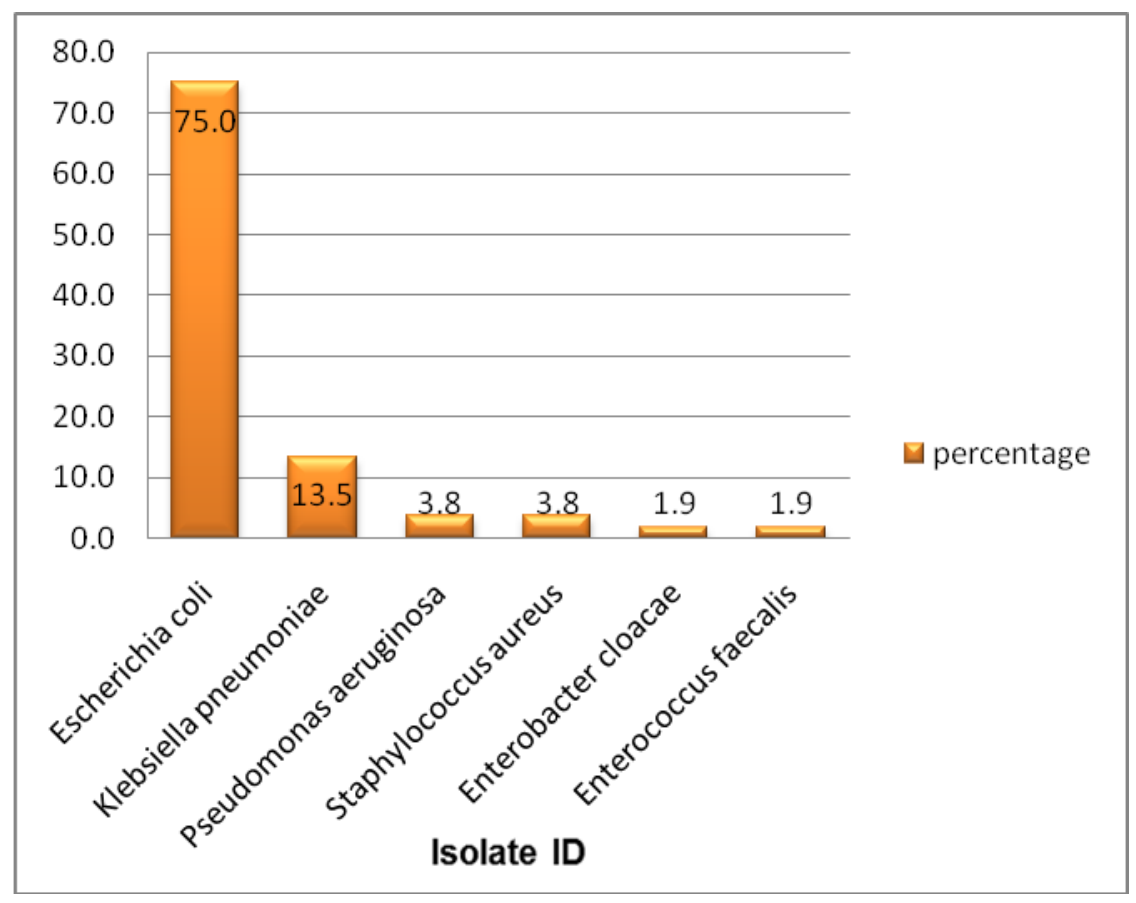


Fig.3 Antimicrobial resistance pattern of gram-negative bacterial isolates from patients with urosepsis

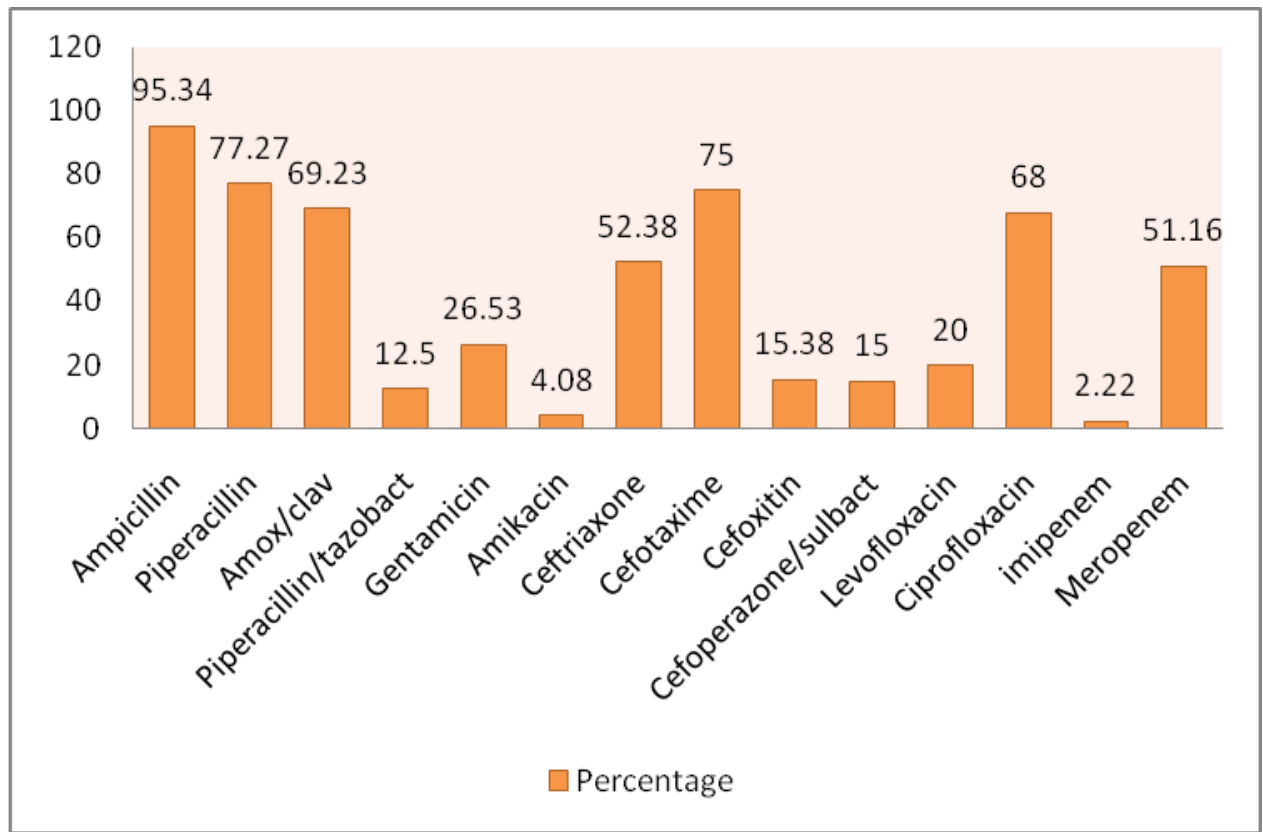

Fig.4 Distribution of 26 multidrug resistant gram-negative bacteria among urosepsis isolates

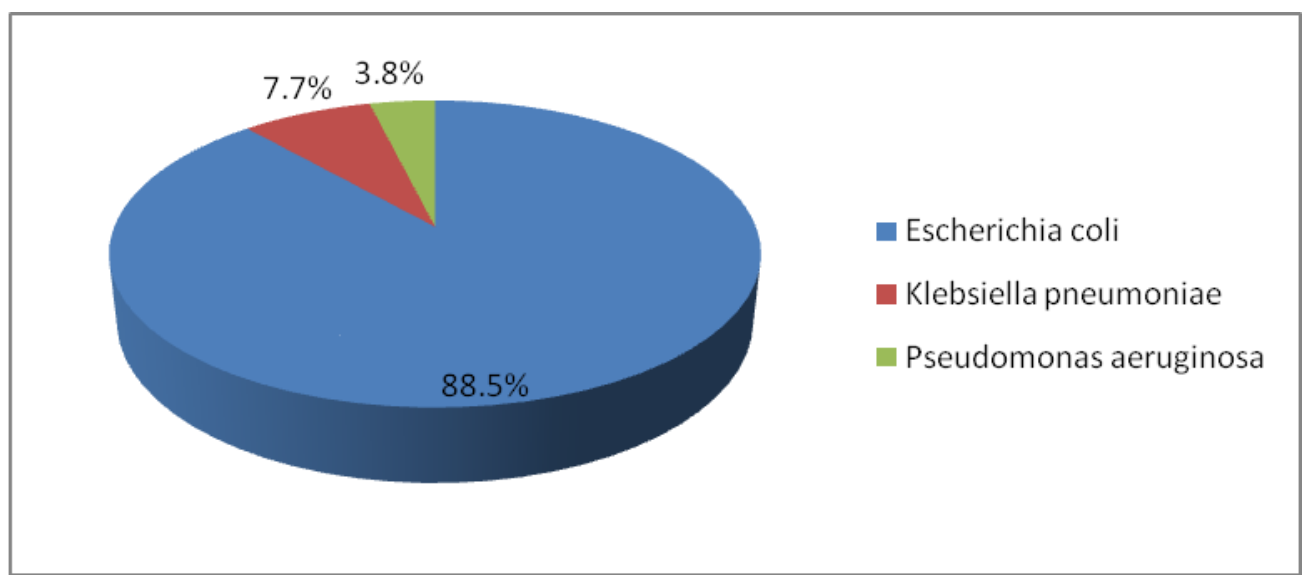

High percentage of isolates from patients with urosepsis, are prone to infections by multidrug resistant strains, due to recurrent infections in the past and co morbid conditions leading to prolonged hospitalization. ESBL production was found to be a factor precluding the use of cephalosporins and other betalactams as well as aminopenicillins. ESBL producing organisms have shown to cause fulminant infection leading to multi organ failure by
Krueger et al., 2007. None of our patients developed any such complication. Combination therapy with imipenem and amikacin would be most appropriate for treatment of patients with urosepsis as noted in the present study. A similar advocacy was noted in the study by Kalra and Raizada, 2009.

Gram-positive organisms, being few in number pose less challenge. However 
Enterococcus faecalis with resistance to several antibiotics would pose a challenge to treatment leaving the choice to vancomycin and linezolid along with aminoglycosides, which have to be given with caution due to renal injury.

Early detection, appropriate treatment and correcting underlying dysfunctions of urinary tract in patients with complicated UTI would decrease the incidence of urosepsis. Type 2 diabetes being detected in epidemic proportions with kidney injury as a complication, patients must be made aware of measures to prevent UTI and seek early intervention to avoid urosepsis.

In conclusion, urosepsis is a life threatening complication of UTI with mortality rates as high as $30 \%-40 \%$. Early recognition of symptoms followed by accurate diagnosis and early specific therapy is essential to decrease morbidity and mortality. Comprehensive management with timely inputs from microbiologists, surgeons, physicians and radiologists is essential for a favorable outcome. Patients with existing co morbid conditions must be educated on the possible risk of UTI leading to urosepsis. They must be advised to seek early intervention in order to avoid poor outcome. With the background knowledge of bacterial spectrum and antibiogram pattern among patients with urosepsis, it would be helpful in starting empiric treatment, as well to formulate an antibiotic policy appropriate for treatment of these cases.

\section{Limitation}

A larger sample size would have helped us to get conclusive evidence of risk factors for urosepsis. Greater number of bacterial isolates would have given a better representative data of antibiogram for formulating antibiotic policy and empiric treatment of urosepsis in the hospital.

\section{Acknowledgement}

Special thanks to ICMR for granting the fund for this ICMR STS 2015 project.

\section{References}

Cardoso, T., Ribeiro, O., Costa-Pereira, A., Carneiro, A. 2008. Communityacquired and healthcare-related urosepsis: A multicenter prospective study. Crit. Care, 12: 18.

Chen, L.F., Chiu, C., Lo, J., Tsai, S., Weng, L., Anderson, D. et al. 2013. Clinical characteristics and antimicrobial susceptibility pattern of hospitalized patients with community acquired urinary tract infections at a regional hospital in Taiwan. Health Infect. Dec., 19(1): 20-25.

CLSI. 2015. Performance Standards for Antimicrobial Susceptibility Testing; Twenty-Fourth Informational Supplement. CLSI document M100S24. Wayne, PA: Clinical and Laboratory Standards Institute.

Garg, V., Bose, A., Jindal, J., Goyal, A. 2015. Comparison of clinical presentation and risk factors in diabetic and non-diabetic females with urinary tract infection assessed as per the European Association of Urology Classification. J. Clin. Diagn. Res., 9(6): 12-14.

Geerlings, S.E., Stolk, R.P., Camps, M.J., Netten, P.M., Hoekstra, J.B., Bouter, K.P., Bravenboer, B., Collet, J.T., Jansz, A.R., Hoepelman, A.I. 2000. Asymptomatic bacteriuria may be considered a complication in women with diabetes. Diabetes Care, 23(6): 744-749.

Gosciniak, M., Kawecki, D., Miklaszewska, M., Truszewski, Z., Lazowski, T., Wielgos, M. and Radziszewski. 2014. Fatal Urosepsis: A 41 Year-Old 
Pregnant Woman - Case Report. Open J. Urol., 4: 137-141.

Hofmann, W. 1990. Urosepsis and uroseptic shock. Z. Urol. Nephrol., 83: 317-324.

Kalra, O.P., Raizada, A. 2009. Approach to a patient with urosepsis. J. Glob. Infect. Dis., 1(1): 57-63.

Krueger, W.A., Unertl, K.E., Kempf, V.A.J., Schroeder, T.H. 2007. Case Report: Treatment of recurrent urosepsis caused by ESBL-producing Escherichia coli with tigecycline. JCM, 1: 1-12.

Lipsky, B.A. 1989. Urinary tract infections in men. Epidemiology, pathophysiology, diagnosis, and treatment. Ann. Intern. Med., 110: $138-150$.

Magiorakos, A.P., Srinivasan, A., Carey, R.B., Carmeli, Y., Falagas, M.E., Giske, C.G. 2012. Multidrug-resistant, extensively drug-resistant and pandrug-resistant bacteria: an international expert proposal for interim standard definitions for acquired resistance. Clin. Microbiol. Infect., 18: 268-281.

McNally, A., Alhashash, F., Collins, M., Alqasim, A., Paszckiewicz, Weston, V., Diggle, M. 2013. Genomic analysis of extra-intestinal pathogenic Escherichia coli urosepsis. Clin. Microbiol. Infect., 19(8): 328-334.

Pradipta, I.S., Sodik, A.C., Lestari, K., Halimah, E., Diantini, A., Abdulah, R. 2013. Antibiotic resistance in sepsis patients: evaluation and recommendation of antibiotic use. $N$.
Am. J. Med. Sci., 5(6): 344-352.

Schweizer, M.L., Furuno, J.P., Harris, A.D., Johnson, J.K., Shardell, M.D., McGregor, J.C. 2011. Comparative effectiveness of nafcillin or cefazolin versus vancomycin in methicillinsusceptible Staphylococcus aureus bacteremia. BMC Infect. Dis., 11: 279283.

Sorensen, S.M., Schonheyder, H.C., Nielsen, H. 2013. The role of imaging of the urinary tract in patients with urosepsis. Int J. Infect. Dis., 17(5): 299-303.

Sugimoto, K., Adomi, S., Koike, H., Esa, A. 2013. Praocalcitonin as an indicator of urosepsis. Res. Rep. Urol., 5: 77-80.

Tal., S., Guller, V., Levi, S., Bardenstein, R., Berger, D., Gurevich, I., Gurevich, A. 2005. Profile and prognosis of febrile elderly patients with bacteremic urinary tract infection. J. Infect., 50(4): 296-305.

Wagenlehner, F.M., Pilatz, A., Naber, K.G., Weidner, W. 2008. Therapeutic challenges of urosepsis. Eur. J. Clin. Invest., 38(S2): 45-49.

Wagenlehner, F.M., Weidner, W., Lichtenstern, C., Weigand, M.A., Rolfes, C., Mayer, K., Weigand, M.A. 2013. Diagnosis and management for urosepsis. Int. J. Urol., 20: 963-970.

Wagenlehner, F.M., Weidner, W., Naber, K.G. 2007. Optimal management of urosepsis from the urological perspective. Int. J. Antimicrob. Agents, 30(5): 390-397.

\section{How to cite this article:}

Martha Rinnah Bijou, K. Sandhya Bhat and Reba Kanungo. 2016. Characteristics of Blood Stream Isolates in Urosepsis from a Tertiary Care Hospital. Int.J.Curr.Microbiol.App.Sci. 5(10): 424-431. doi: http://dx.doi.org/10.20546/ijcmas.2016.510.048 